\title{
Computational Analysis on Longitudinal Equivalent Bending Stiffness under the Influence of Shield-tunnel Transverse Deformation
}

\author{
Ming-Yu LI ${ }^{1, a,{ }^{*}}$, Zhang-Jun HUANG ${ }^{2, b}$, Yan-Wei ZHANG ${ }^{1, c}$, Xiao-Fei BIAN ${ }^{1, d}$ \\ ${ }^{1}$ Zhengzhou University, Henan Zhengzhou, 450001, China \\ ${ }^{2}$ ERCHU CO., LTD. of China Railway Tunnel Group, Hebei Sanhe, 065200, China \\ awudizhenjime@126.com, b812409787@qq.com, ${ }^{\text {c3 } 347005051 @ q q . c o m, ~ d 1170005760 @ q q . c o m ~}$ \\ ${ }^{*}$ Corresponding author
}

Keywords: Shield Tunnel, Convergence Deformation, Longitudinal Equivalent Bending Stiffness, Elliptic Equation.

\begin{abstract}
In order to study the lining longitudinal deformation rule under the influence of the lining transverse deformation in the process of long-term settlement of operating metro shield tunnel with straight joint, based on the field test monitoring data of Tibet Road-Zhongxing Road shield tunnel in the M8 of shanghai, and considering the tunnel convergence, this problem is studied by amending equivalent continuous model. Then there is a comparison between the existing theoretical methods and this calculation method. The results showed that, in the ring joints, no considering shearing, the maximum of ring joint open, the tension and compression stress of segment, and bolt stress slightly decrease with the increase of deformation of tunnel convergence under the bending. The smaller the curvature radius of the shield-tunnel longitudinal deformation is, the larger the ring joint open under the influence of tunnel convergence is.
\end{abstract}

\section{Introduction}

Due to the joint, the stiffness of the lining is reduced in the shield tunnel with straight joint. When the longitudinal differential settlement occurs, there is joint staggering and joint opening between the rings. These will cause the joint leakage, the segment crack and track irregularity, etc. endangering the safety of operational tunnel. Therefore, to ensure the safety of operational tunnel, it is very important of the deformation and force characteristics of the ring joint longitudinally along tunnel. But so far, this research is still at a preliminary stage. In the process of long-term settlement of operational tunnel, the transverse deformation and the longitudinal deformation often occur simultaneously with the longitudinal differential settlement increasing. For the reason, the influence of tunnel convergence should be considered when analyzing the longitudinal deformation and stress of the ring with straight joint. The longitudinal equivalent bending stiffness model was studied in the references[1, 2, 3, 4], but without having considered the relationship between the transverse deformation and the longitudinal deformation. To sum up, with considering the relationship between the transverse deformation and the longitudinal deformation, a new longitudinal equivalent bending stiffness model is put forward for interpreting the longitudinal deformation and force of shield tunnel with straight joint more accurately, and providing more valuable theoretical reference for the operational safety jugement of metro tunnel.

\section{Field Measurement Test}

\section{Summary}

The Tibet road $\sim$ Zhongxing Road Tunnel of Shanghai metro line No.8 is $1226 \mathrm{~m}$. It is mainly through Gray muddy clay and Gray clay. The joint segment is used in the shield tunnel. Each lining ring contains a key block, two adjacent blocks, two standard blocks, and an arch bottom block. The design strength grade of segment concrete is C55. The joint bolt is M30. The elastic gasket used for joint waterproofing is composed of water swelling rubber and EPDM. The main design parameters are shown in table 1. 
Tab.1 The Main Design Parameters

\begin{tabular}{cc|cc}
\hline The segment & Value & The bolt & Value \\
\hline Thickness $(\mathrm{m})$ & 0.35 & diameter(m) & 0.03 \\
Width $(\mathrm{m})$ & 1 & The number of Ring-joint bolts & 17 \\
Outer diameter $(\mathrm{m})$ & 6.2 & The number of Longitudinal-joint bolts & 12 \\
Inner diameter $(\mathrm{m})$ & 5.5 & The tensile yield strength (MPa) & 640 \\
Ring-joint initial deformation $(\mathrm{m})$ & 0 & The elastic modulus of bolt (GPa) & 206 \\
The design value of the concrete compressive & 26 & length(m) & 0.4 \\
strength (MPa) & & & \\
The design value of the concrete tensile & & & \\
strength (MPa) & 21 & & \\
The elastic modulus of concrete (MPa) & 35500 &
\end{tabular}

The field measurement test section is near the work well, standard section, and the connected aisle respectively, as shown in figure 1. Monitoring projects includ the tunnel convergence and the joint opening. Monitoring period is one year. The monitoring frequency is 3 months.

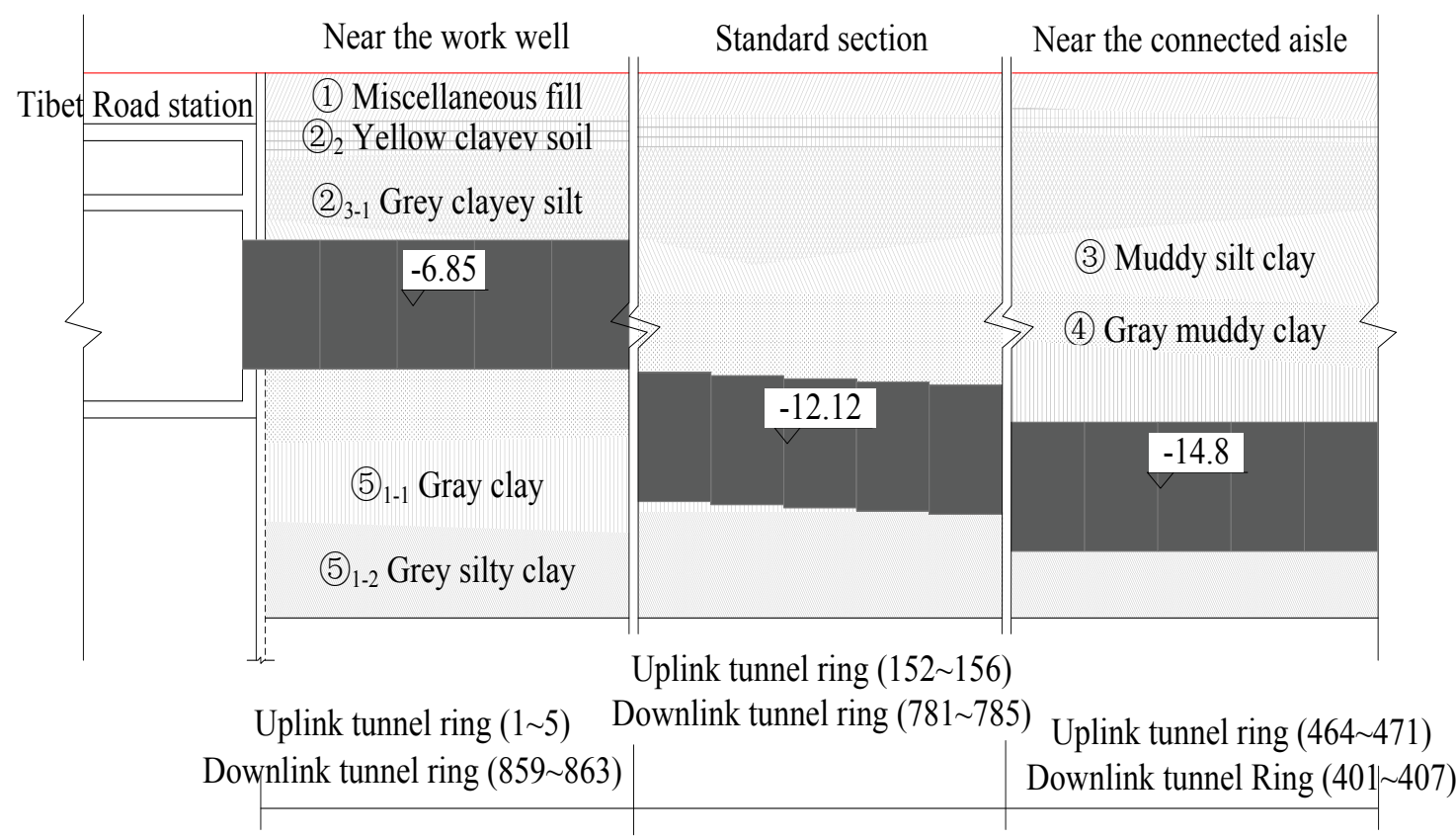

Fig. 1 Schematic Diagram of Monitoring

Tab. 2 Number of Points (tunnel convergence)

\begin{tabular}{|c|c|c|c|c|}
\hline \multirow{2}{*}{ Monitoring area } & \multicolumn{2}{|c|}{ Uplink tunnel } & \multicolumn{2}{|c|}{ Downlink tunnel } \\
\hline & Ring number & Point number & Ring number & Point number \\
\hline \multirow{5}{*}{$\begin{array}{c}\text { Near the work well of } \\
\text { Tibet Road }\end{array}$} & 1 & $\mathrm{XZ1}$ & 863 & $\mathrm{xZ} 30$ \\
\hline & 2 & $\mathrm{xz} 2$ & 862 & $x \geq 29$ \\
\hline & 3 & $\mathrm{xZ3}$ & 861 & $\mathrm{xZ28}$ \\
\hline & 4 & $\mathrm{XZ4}$ & 860 & $\mathrm{xZ} 27$ \\
\hline & 5 & $\mathrm{xZ5}$ & 859 & $x \geq 26$ \\
\hline \multirow{5}{*}{ Standard section } & 152 & & 785 & \\
\hline & 153 & $\mathrm{xz} 6$ & 784 & $\mathrm{xZ} 25$ \\
\hline & 154 & $\mathrm{XZ7}$ & 783 & $\mathrm{XZ24}$ \\
\hline & 155 & & 782 & \\
\hline & 156 & & 781 & \\
\hline \multirow{8}{*}{ Near the connected aisle } & 464 & $\mathrm{xz} 8$ & 408 & $\mathrm{XZ23}$ \\
\hline & 465 & $\mathrm{XZ9}$ & 407 & $\mathrm{xZ} 22$ \\
\hline & 466 & $\mathrm{XZ10}$ & 406 & $\mathrm{XZ21}$ \\
\hline & 467 & $\mathrm{XZ11}$ & 405 & $\mathrm{XZ20}$ \\
\hline & 468 & $\mathrm{XZ12}$ & 404 & XZ19 \\
\hline & 469 & $\mathrm{XZ13}$ & 403 & $\mathrm{XZ18}$ \\
\hline & 470 & $\mathrm{XZ14}$ & 402 & $\mathrm{XZ17}$ \\
\hline & 471 & $\mathrm{XZ15}$ & 401 & $\mathrm{XZ16}$ \\
\hline
\end{tabular}


The initial coordinates is obtained by scaning fifty monitoring points on the ring with the total station. Then, fitting the initial coordinates gets the tunnel convergence by using for references[5,6,7,8,9]. The crack meter is used to survey the ring-joint opening between ring 783 and ring 784. One of crack meters is located in the key block, another is located in the standard block. The number of monitoring points is shown in table 2 .

\section{Data Analysis}

With time increasing, the vertical compression deformation of tunnel increase gradually, as shown in figure 2.

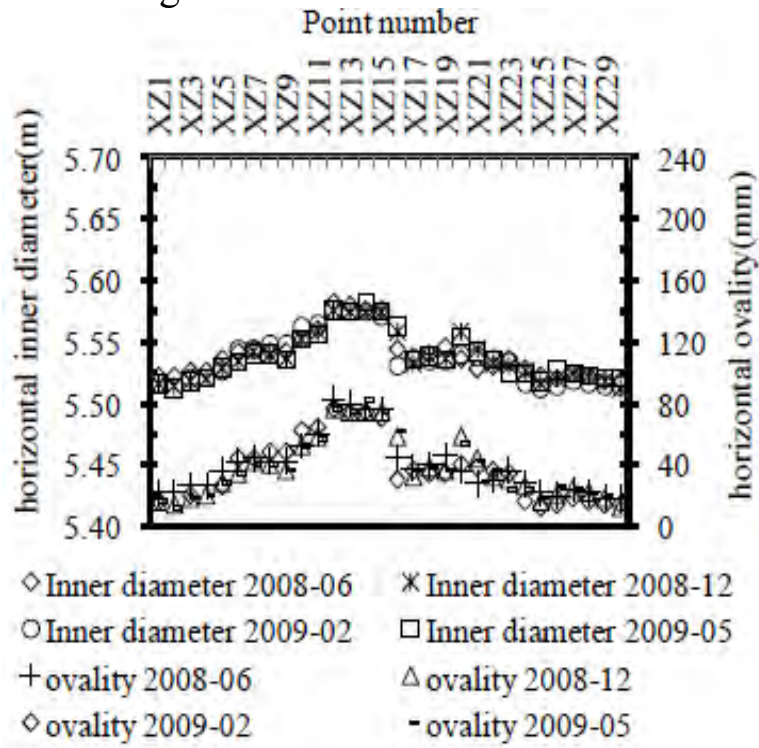

(a)Horizontal

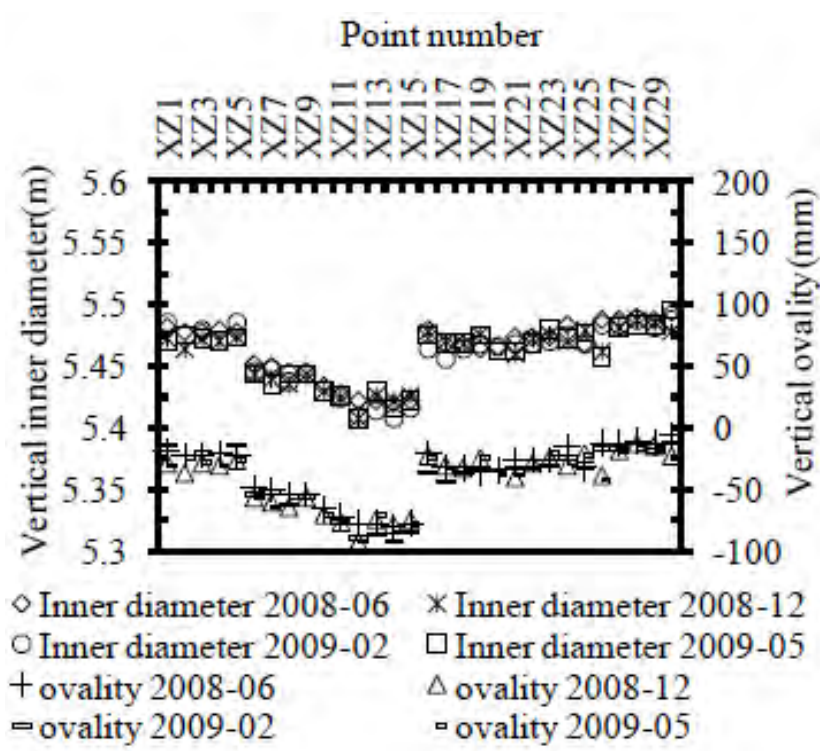

(b) Vertical

Fig.2 Distribution Curve of Tunnel Convergence

Because the tunnel convergence is constrained by the work well and the soil around the tunnel is reinforced when the shield machine got into or out of the work well, the tunnel convergence is relatively smaller near the work well. The soil around tunnel was disturbed in the tunneling press. Then with the connected aisle construction and the operating train vibration, the disturbed soil has been disturbed again. Compared to the other test section, the consolidation deformation of multiple disturbed soil is larger and the consolidation time is longer near the connected aisle. So that the tunnel convergence is relatively larger near here.

\section{Correction Calculation of Longitudinal Equivalent Bending Stiffness}

\section{Model Calculation Assumes}

According to the reference[2], the relative rotation will occur between the two adjacent linings. The relative angle is $\theta$. In theory, the curvature is $\theta / l_{\mathrm{s}}$ when the beam bending. The width of the segment is $l_{\mathrm{s}}$ To solve the relationship of the bending moment and the curvature, the computational assumptions are as follows. The first is plane cross-section assumption. The lining ring is the homogeneous ring. On the cross section, the deformation of every point is proportional to the distance from the neutral axis. The second is not considering the joint staggering. The third, the bolt will bear all tension in the tension zone and the segment will bear all pressure in the compressive zone, when the relative rotation of the two adjacent linings appears in the joint. The fourth, to simplify the calculation, springs evenly distributed in the lining ring is instead of bolts.

$$
K_{r}=\frac{E_{b} A_{b}}{2 \pi r l_{b}}
$$


In the formula (1), the average line stiffness of the bolt is $K_{\mathrm{r}}(\mathrm{kN} / \mathrm{m})$. The elastic modulus of the bolt is $E_{\mathrm{b}}(\mathrm{MPa})$. The cross-sectional area of the bolt is $A_{\mathrm{b}}\left(\mathrm{m}^{2}\right), A_{\mathrm{b}}=\pi d_{\mathrm{b}}{ }^{2} / 4$. The diameter of the bolt is $d_{\mathrm{b}}(\mathrm{m})$. The length of the bolt is $l_{\mathrm{b}}(\mathrm{m})$. When the cross section in the elastic state, the stress of all bolts is less than $P_{\mathrm{y}}$. The pretightening force of the bolt is $P_{\mathrm{y}}$. The analysis of unit stress and strain is shown in figure 3 . The relationship between the moment and curvature of the calculating unit is obtained according to the deformation compatibility condition and force equilibrium relationship.

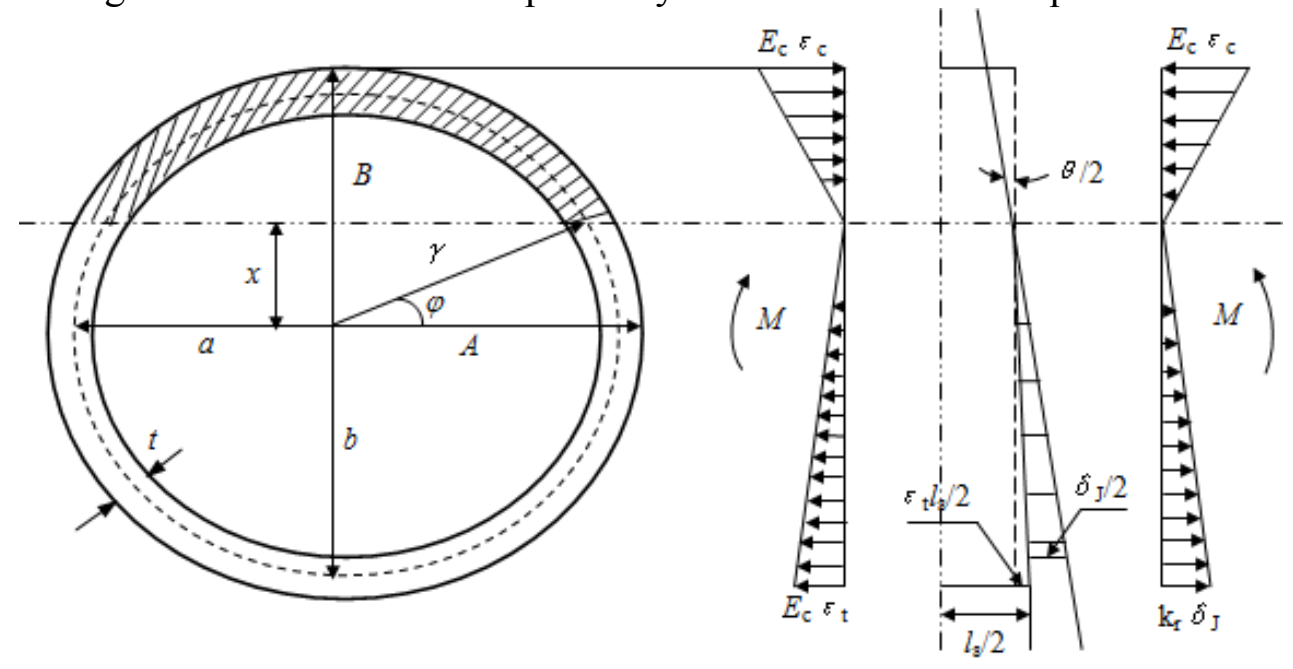

Fig.3 Diagram of Elastic Stress and Strain

\section{Calculation Process}

The deformation compatibility condition is as follows:

$$
\begin{aligned}
& \varepsilon_{c} \frac{l_{s}}{2}=(B-x) \frac{\theta}{2} \\
& \frac{b+x}{B+x} \varepsilon_{t} \frac{l_{s}}{2}+\frac{\delta_{J}}{2}=(b+x) \frac{\theta}{2}
\end{aligned}
$$

In the formula (2) and formula (3), the semi-minor axis of elliptical lining ring is $B(\mathrm{~m}), B=b+t / 2$. The long and short radius of elliptical lining ring respectively is $a$ and $b(\mathrm{~m}), a=(t+d+\Delta \mathrm{D} l) / 2, b=(t+d+$ $\left.\Delta_{\mathrm{Dv}}\right) / 2$. The inner and outer diameter of the tunnel respectively is $d$ and $D(\mathrm{~m})$. the vertical and horizontal convergence of the tunnel is $\Delta_{\mathrm{D} l}$ and $\Delta_{\mathrm{Dv}}(\mathrm{mm}), \Delta_{\mathrm{D} l}, \Delta_{\mathrm{Dv}}>0$. The maximum deformation of bolt is $\delta_{\mathrm{J}}(\mathrm{mm})$. The width of the lining ring is $t(\mathrm{~m})$. The distance from the computing unit to the neutral axis is $x(\mathrm{~m}) . \varphi$ is the angle between the straight line of the computing unit and the neutral axis. $\varphi=\sin ^{-1}(x / r)$. The tensile and compressive strain of the segment respectively is $\varepsilon_{\mathrm{t}}$ and $\varepsilon_{\mathrm{c}}$.

The force equilibrium relationship is as follows:

$$
\begin{aligned}
& 2 \frac{E_{c} \varepsilon_{c}}{B-x} \int_{\varphi}^{\pi / 2}(r \sin \alpha-x) r t d \alpha=2 \frac{E_{c} \varepsilon_{t}}{B+x} \int_{-\pi / 2}^{\varphi}(-r \sin \alpha+x) r t d \alpha=2 \frac{K_{r} \delta_{J}}{b+x} \int_{-\pi / 2}^{\varphi}(-r \sin \alpha+x) r d \alpha \\
& 2 \frac{E_{c} \varepsilon_{c}}{B-x} \int_{\varphi}^{\pi / 2}(r \sin \alpha-x)^{2} r t d \alpha+2 \frac{E_{c} \varepsilon_{t}}{B+x} \int_{-\pi / 2}^{\varphi}(-r \sin \alpha+x)^{2} r t d \alpha=M
\end{aligned}
$$

In the formula (4) and formula (5), The distance from the computing unit to the centroid is $r(\mathrm{~m})$, $r=b / \sqrt{1-\varepsilon^{2} \cos ^{2} \alpha}$. The elastic modulus of the segment is $E_{\mathrm{c}}(\mathrm{MPa})$; The ellipse center rate is $\varepsilon$, $\varepsilon=\sqrt{1-b^{2} / a^{2}}$. The bending moment is $\mathrm{M}(\mathrm{kN} \cdot \mathrm{m})$.

The solution of simultaneous equations from the formula (1) to formula (5) is as follows: 


$$
\theta=\frac{M l_{s}}{E_{c} t b^{3} K}
$$

$$
K=\frac{\pi}{6}+\frac{\frac{\pi}{2}+\varphi}{3} \frac{8 \sqrt{2}-4 \sqrt{2} \varepsilon^{2}-4 \sqrt{2} \varepsilon^{2} \sin \varphi-6 \sqrt{2} \varepsilon^{2} \cos ^{2} \varphi}{\left(2-\varepsilon^{2}-\varepsilon^{2} \sin \varphi\right)^{\frac{5}{2}}}+\frac{\frac{\pi}{2}-\varphi}{3} \frac{8 \sqrt{2}-4 \sqrt{2} \varepsilon^{2}+4 \sqrt{2} \varepsilon^{2} \sin \varphi-6 \sqrt{2} \varepsilon^{2} \cos ^{2} \varphi}{\left(2-\varepsilon^{2}+\varepsilon^{2} \sin \varphi\right)^{\frac{5}{2}}}+\frac{\pi}{6}
$$$$
\frac{1-\varepsilon^{2} \cos ^{2} \varphi-3 \varepsilon^{2} \sin ^{2} \varphi \cos ^{2} \varphi}{\left(1-\varepsilon^{2} \cos ^{2} \varphi\right)^{\frac{5}{2}}}-\frac{\sin \varphi}{\sqrt{1-\varepsilon^{2} \cos ^{2} \varphi}} \frac{\lambda}{\beta}\left(\frac{\sin \varphi}{\varepsilon \sqrt{1-\varepsilon^{2} \cos ^{2} \varphi}} \ln \frac{1+\varepsilon \cos \varphi}{1-\varepsilon \cos \varphi}+\frac{\frac{\pi}{2}+\varphi}{6}\left(\frac{1-3 \varepsilon^{2} \cos ^{2} \varphi+2 \varepsilon^{2} \cos ^{4} \varphi}{\left(1-\varepsilon^{2} \cos ^{2} \varphi\right)^{\frac{5}{2}}}\right.\right.
$$$$
\left.\left.+8 \sqrt{2} \frac{2-\varepsilon^{2}-\varepsilon^{2} \sin \varphi-\varepsilon^{2} \cos ^{2} \varphi}{\left(2-\varepsilon^{2}-\varepsilon^{2} \sin \varphi\right)^{\frac{5}{2}}}+1\right)-\frac{\sin \varphi \cos \varphi}{\left(1-\varepsilon^{2} \cos ^{2} \varphi\right)^{\frac{3}{2}}}\right)
$$

$\lambda=\frac{\pi}{3}\left(1+\frac{1}{\sqrt{1-\varepsilon^{2} \cos ^{2} \varphi}}\right)+\frac{4 \sqrt{2}}{3}\left(\frac{\frac{\pi}{2}-\varphi}{\sqrt{2-\varepsilon^{2}+\varepsilon^{2} \sin \varphi}}+\frac{\frac{\pi}{2}+\varphi}{\sqrt{2-\varepsilon^{2}-\varepsilon^{2} \sin \varphi}}\right)$

$\beta=\frac{1}{\varepsilon} \ln \frac{1+\varepsilon \cos \varphi}{1-\varepsilon \cos \varphi}+\frac{\pi / 2+\varphi}{3} \frac{\sin \varphi}{\sqrt{1-\varepsilon^{2} \cos ^{2} \varphi}}\left(1+\frac{4 \sqrt{2}}{\sqrt{2-\varepsilon^{2}-\varepsilon^{2} \sin \varphi}}+\frac{1}{\sqrt{1-\varepsilon^{2} \cos ^{2} \varphi}}\right)$.

With the equivalent continuous beam bending, The formula of angle $\theta_{\text {eq }}$ is as follows:

$$
\theta_{e q}=\frac{M l_{s}}{(E I)_{e q}}
$$

By comparing formula (6) with formula (7), the equivalent elastic bending rigidity of shield tunnel with straight joint $(E I)_{\mathrm{eq}}$ is as follows:

$$
(E I)_{e q}=E_{c} t b^{3} K
$$

The Elastic limit bending moment $\mathrm{M}_{\mathrm{y}}(\mathrm{kN} \cdot \mathrm{m})$ of the unit is as follows:

$$
\begin{aligned}
& \sigma_{y}=\frac{M_{y}}{(E I)_{e q}} b\left(\frac{\sin \varphi}{\sqrt{1-\varepsilon^{2} \cos ^{2} \varphi}}+1\right)=\frac{N_{y}}{(E A)_{e q}^{T}} \\
& M_{y}=\frac{N_{y}(E I)_{e q}}{b\left(\frac{\sin \varphi}{\sqrt{1-\varepsilon^{2} \cos ^{2} \varphi}}+1\right)(E A)_{e q}^{T}}
\end{aligned}
$$

The solution of simultaneous equations from the formula (2) to formula (6) is as follows:

$$
\varsigma E_{s} \frac{\pi d_{b}^{2}}{4 l_{b}}=\frac{E_{c} t}{n l_{\mathrm{s}}} \frac{\frac{\pi}{3} a\left(1+4 \sqrt{1-\frac{\varepsilon^{2}}{2}}+\sqrt{1-\varepsilon^{2}}\right)}{\beta / \eta-1}
$$

In the formula (11), $\eta=\frac{1}{\varepsilon} \ln \frac{1+\varepsilon \cos \varphi}{1-\varepsilon \cos \varphi}-\frac{\pi / 2-\varphi}{3} \frac{\sin \varphi}{\sqrt{1-\varepsilon^{2} \cos ^{2} \varphi}}\left(1+\frac{4 \sqrt{2}}{\sqrt{2-\varepsilon^{2}+\varepsilon^{2} \sin \varphi}}+\frac{1}{\sqrt{1-\varepsilon^{2} \cos ^{2} \varphi}}\right)$.

The maximum compressive stress of the segment $\sigma_{\mathrm{c}}(\mathrm{MPa})$ is as follows:

$$
\sigma_{c}=\frac{E_{c}}{\rho}\left(b+\frac{t}{2}-\frac{b \sin \varphi}{\sqrt{1-\varepsilon^{2} \cos ^{2} \varphi}}\right)
$$

The maximum tensile stress of the bolt $\sigma \mathrm{t}(\mathrm{MPa})$ is as follows: 


$$
\sigma_{t}=\frac{E_{c}}{\rho}\left(1-\frac{\sin \varphi}{\sqrt{1-\varepsilon^{2} \cos ^{2} \varphi}} \frac{\lambda}{\beta}\right)\left(b+\frac{t}{2}+\frac{b \sin \varphi}{\sqrt{1-\varepsilon^{2} \cos ^{2} \varphi}}\right)
$$

The maximum tensile stress of the bolt fT $(\mathrm{kPa})$ is as follows:

$$
f_{T}==\varsigma E_{s} \frac{l_{s}}{\rho l_{b}} \frac{\sin \varphi}{\sqrt{1-\varepsilon^{2} \cos ^{2} \varphi}} \frac{\lambda}{\beta}\left(b+\frac{b \sin \varphi}{\sqrt{1-\varepsilon^{2} \cos ^{2} \varphi}}\right)
$$

The maximum opening of the joint $\delta \mathrm{T}$ is as follows:

$$
\delta_{T}=\delta_{J}+\delta_{0}=\frac{l}{\rho} \frac{\sin \varphi}{\sqrt{1-\varepsilon^{2} \cos ^{2} \varphi}} \frac{\lambda}{\beta}\left(b+\frac{b \sin \varphi}{\sqrt{1-\varepsilon^{2} \cos ^{2} \varphi}}\right)+\delta_{0}
$$

In the above formula, the curture raduis is $\rho(\mathrm{m})$. The initial joint opening of the ring is $\delta_{0}(\mathrm{~mm})$. It is caused by assembling the segment in the process of construction. $\zeta$ is the reduction coefficient of the elastic modulus of the bolt sheared in the bolt hole, $0<\zeta<1$.

\section{Example Analysis}

In field test, the minimum longitudinal deformation curvature radius of the tunnel is $\rho$ min, $\rho$ min $=3.33 \times 105 \mathrm{~m}$. Axial tensile deformation is $0.079 \mathrm{~mm} . \delta 0=0, \zeta=1$. For the ring-joint opening, the monitoring data and caculation results are compared in table 3 . The comparison results is almost the same. It shows that the calculation method can reflect accurately the longitudinal deformation characteristic of shield tunnel with the straight joint.

Tab. 3 Comparative Analysis of the Ring-joint Opening

\begin{tabular}{cccc}
\hline & Key block & Standard block & Arch bottom block \\
\hline Field measurement test $(\mathrm{mm})$ & 0.079 & 0.096 & 0.101 \\
This method $(\mathrm{mm})$ & 0.079 & 0.086 & 0.097 \\
\hline
\end{tabular}

\section{Parameter Analysis}

Analyzed parameters includ the tunnel convergence, the curvature radius, the tensile and compressive stress of the segment, the tensile stress, and the ring-joint opening, as shown from figure 4 to figure 7 . For the pure bending tunnel, the ring-joint opening and the internal force of the segment and the bolt slightly reduce with With the increase of the tunnel convergence. When the longitudinal curvature radius of the tunnel is small, the tunnel convergence has an obvious effect on the internal force of the segment and the bolt.

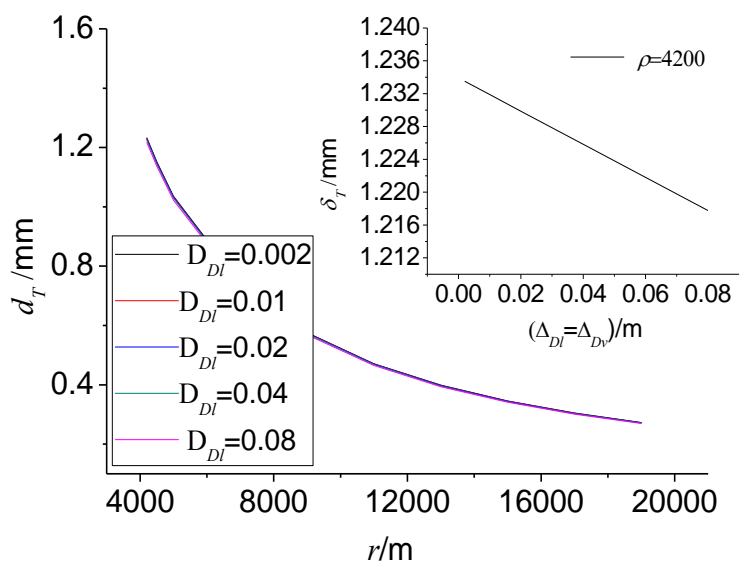

Fig.4 The Curve of Ring Joint Open and Tunnel Convergence

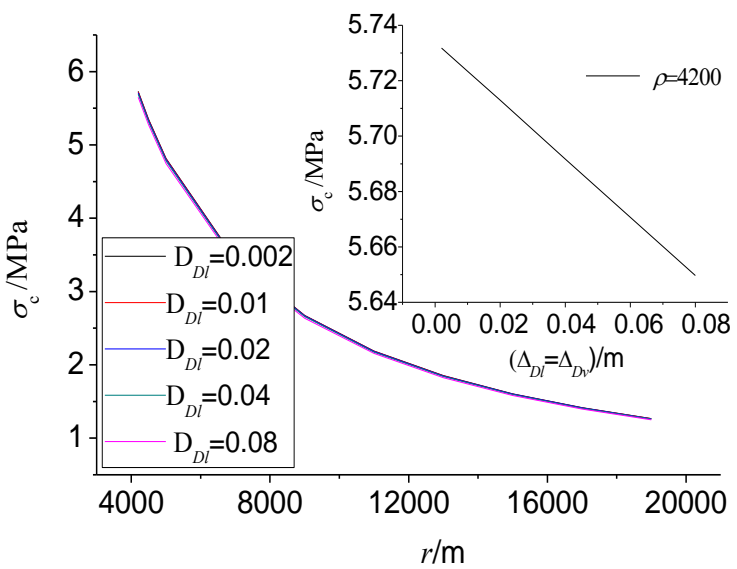

Fig.5 The Curve of Segment Stress and Tunnel Convergence 


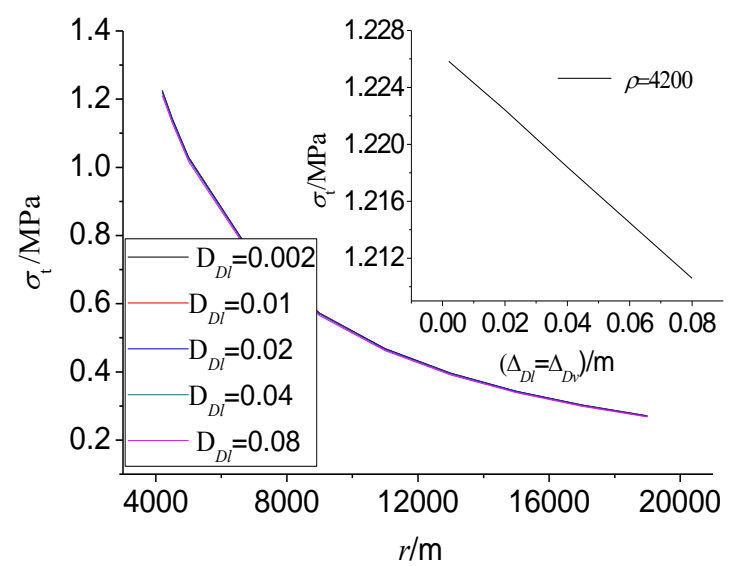

Fig. 6 The Curve of Segment Tensile Stress and Tunnel Convergence

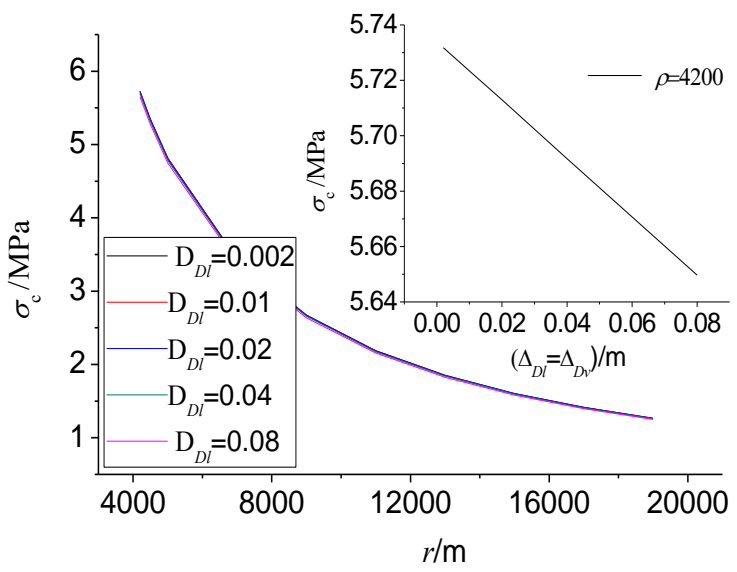

Fig. 7 The Curve of Longitudinal Tensile Stress in Bolts and Tunnel Convergence

\section{Comparison and Analysis}

In the analysis and calculation, according to the code for construction and acceptance of shield tunnel (GB50446-2008), $\rho_{\min }=15000 \mathrm{~m}, \mathrm{f}_{\mathrm{T}}=6.4 \times 10^{5} \mathrm{kPa}, \Delta_{\mathrm{Dl}}=\Delta_{\mathrm{Dv}}=31 \mathrm{~mm}, \delta_{0}=0, \zeta=1$. Comparing this method with the method of references[2,3,4], the analysis result is as shown in table 4 .

When the longitudinal curvature of the shiled tunnel with the straight joint is the same, the comparative analysis of the ring-joint opening: the reference[4] $<$ this article $<$ the reference[3] $<$ the reference[2]; the comparative analysis of the tensile stress of the bolt: this article $<$ the reference[3] $<$ the reference[2] $<$ the reference[4].

When the maximum tensile stress of the bolt is the same, the comparative analysis of the ring-joint opening: the reference[4] $<$ the reference[3] $<$ this article $<$ the reference[2]; the comparative analysis of the longitudinal curvature of the tunnel: the reference[2] $<$ this article $<$ the reference[3] $<$ the reference[4].

Tab. 4 Comparison Table

\begin{tabular}{llll}
\hline Acceptance criteria & Differential calculation methods & $\delta \mathrm{T}(\mathrm{mm})$ & $\mathrm{fT}(105 \mathrm{kPa})$ \\
\hline \multirow{3}{*}{$\rho$ min=15000m } & This method & 0.34 & 1.77 \\
& Reference[2] & 0.4 & 3.84 \\
& Reference[3] & 0.35 & 2.48 \\
& Reference[4] & 0.32 & 3.97 \\
\hline Acceptance criteria & Differential calculation methods & $\rho(\mathrm{m})$ & $\delta \mathrm{T}(\mathrm{mm})$ \\
\hline \multirow{5}{*}{$\mathrm{fT}=6.4 \times 105 \mathrm{kPa}$} & This method & 4149 & 1.24 \\
& Reference[2] & 3193 & 2.06 \\
& Reference[3] & 4682 & 1.11 \\
& Reference[4] & 8694 & 0.55 \\
\hline
\end{tabular}

\section{Conclusion}

With the relationship between the transverse deformation and the longitudinal deformation considered, the existing longitudinal equivalent bending stiffness model is amended in this article. Then, based on the field test monitoring data of Tibet Road-Zhongxing Road shield tunnel in the M8 of shanghai, the analysis results of this method and the referenc $[2,3,4]$ is compared. The main analysis conclusion is as follows:

For the pure bending tunnel, the ring-joint opening and the internal force of the segment and the bolt slightly reduce with With the increase of the tunnel convergence. When the longitudinal 
curvature radius of the tunnel is small, the tunnel convergence has an obvious effect on the internal force of the segment and the bolt.

In the process of long-term settlement of operational tunnel, the transverse deformation and the longitudinal deformation often occur simultaneously with the longitudinal differential settlement increasing. In this article, the deformation and force of the ring joint is only analyzed under the pure bending state. The longitudinal equivalent stiffness model of the shear and tension should be studied in the future research.

\section{References}

[1]Y.G. Lin, S.M. Liao, G.B. Liu, A discussion of the factors effecting on longitudinal deformation of subway tunnel, Underground Space. Vol. 20 (2000) No.4, pp. 264-267. (In Chinese)

[2]Y.D. Ye, Research on deformation and method of health diagnose of operational subway structures in soft soil. (Ph.D., Tongji University, China 2007). (In Chinese)

[3]Y.L. Zheng, W. X. Han, Q.H. Tong, etc., Study on longitudinal crack of shield tunnel segment joint due to asymmetric settlement in soft soil, Chinese journal of rock mechanics and engineering. Vol. 24 (2005) No.24, pp. 4552-4558. (In Chinese)

[4]Z.P. Lu, The safety evaluation research of metro shield tunnel during construction and operation based on static measured data. (Ph.D., Tongji University, China 2008). (In Chinese)

[5]C. Yan, The application of total station in tunnel deformation monitoring. (MS., Tongji University, China 2005). (In Chinese)

[6]W.S.Qiu, Analytic geometry, Peking University press, Beijing, 1999. (In Chinese)

[7]P.L. Rosin, A note on the least squares fitting of ellipses, Pattern recognition letters. (1993) No.14, pp. 799-808.

[8]W. Gander, G.H. Golub, R. Strebel, Least-Square fitting of circles and ellipses, BIT. (1994) No.43, pp. 558-578.

[9]G. Taubin, Estimation of planar curves, surfaces and nonplanar space curves defined by implicit equations with applications to edge and range image segmentation, IEEE trans pattern analysis and machine intelligence. Vol.13, (1991) No.1, pp.1115-1138. 